\title{
Susceptibilidad a la sugestión en función de los factores de personalidad
}

Francisco J. Labrador Universidad Complutense de Madrid.

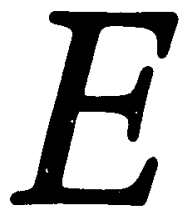

\section{INTRODUCCION}

El siguiente experimento podríamos encuadrarlo dentro de los experimentos dirigidos a comparar la sugestionabilidad diferencial de los sujetos ante informes psicológicos. El trabajo no es nuevo, y el hecho de que los sujetos resultan fuertemente influidos por informes psicológicos generalizados ha sido repetidamente demostrado en la literatura experimental, Ulrich, Stachnik y Staiton (1963), Synder y Larson (1972), Avia y Bragado (1975).

No nos interesa volver a demostrar el hecho de que las personas puedan ser influidas a aceptar un informe generalizado y standard de personalidad, aunque en definitiva nuestros datos deberán corroborarlos si el trabajo se ha realizado correctamente; lo que nos interesa es ver hasta qué punto es posible predecir en base a los modelos de personalidad cuáles son los sujetos más o menos susceptibles a ser influidos por la presentación de estos informes.

Realmente sobre este tema no se han presentado muchos trabajos, al menos que lo traten directamente, siendo uno de los más importantes el anteriormente citado de Avia y Bragado. En este trabajo, los resultados indicaban que sí podía hacerse alguna predicción a partir de las variables de personalidad. En concreto eran las variables de rigidez medidas a través del cuestionario RII, y una variable denominada por los investigadores como «ansiedad facilitadora del rendimiento", las que mejor explicaban las diferencias, si bien, como estas mismas investigadoras comentan, las correlaciones que presentan son de escasa cuantía y además, en el caso de la rigidez, la correlación es de distinto signo para hombres que para mujeres. Pues mientras que en el caso de los hombres la correlación entre rigidez y acuerdo con el informe era positiva, en las mujeres era negativa.

Eysenck (1947), indicaba que la dimensión de $\mathrm{N}$ era importante para distinguir $\mathrm{y}$ diferenciar el grado de sugestibilidad de los sujetos, siendo éstos tanto más sugestionables cuanto mayor fuera su puntuación en N. Es más, aun en contra de lo previsto, indica Eysenck, aparecieron diferencias entre los sujetos histéricos y distímicos, siendo ligeramente más sugestionables los sujetos distímicos. 
Trabajos posteriores como el de Furneaux (1961), no encontraron diferencias importantes en cuanto al grado de sugestionabilidad de los sujetos en función de sus puntuaciones en $\mathbf{N}$, ni tampoco en E-I. Sin embargo, al llevar a cabo un análisis de datos denominado por Furneaux (1961) «análisis de zona», y que nosotros denominamos, de acuerdo con la terminología de Ghiselli (1963), análisis de la "variable moduladora" (de acuerdo con el cual se deberían tener en cuenta las puntuaciones en la dimensión E-I para poder hacer predicciones en base a la dimensión N), encontró que sus nuevas hipótesis, que indicaban que los sujetos con valores altos en $\mathrm{N}$ y $\mathrm{E}$ serían poco sugestionables al igual que los sujetos con puntuaciones bajas en $\mathrm{N}$ y en $\mathrm{E}$, mientras que los sujetos con valores altos en una de estas dimensiones y bajos en la otra, deberían ser mucho más sugestionables, se confirmaban. Resultados muy similares obtuvieron también Furneaux y.Gibson (1961) en un test de hipnosis. Aunque también se han presentado resultados contrarios en Furneaux y Bentler (1963) y Hilgard (1965).

Aunque la base teórica para el desarrollo de la experimentación y formulación de estas nuevas hipótesis, no está suficientemente clara, Eysenck (1966 y 1967), al revisar estos trabajos, apoya estas predicciones como más correctas que las predicciones basadas únicamente en la dimensión de $\mathrm{N}$. Otros trabajos posteriores como los de Gibson y Curran (1974), Gibson y Corcoran (1975) y Gibson, Corcoran y Curran (1977), este último en un diseño más completo y con control del nivel de drive mediante el uso de diazepan, han vuelto a confirmar las predicciones formuladas por Eysenck (1966), y que serán las que definitivamente someteremos a prueba. No obstante, dado que estos trabajos han ido dirigidos a estudiar la relación entre las dimensiones y el grado de susceptibilidad a ser hipnotizados o sugestibilidad al test de balanceo corporal y nuestro trabajo se dirige a ver el grado en que son sugestionables los sujetos a partir de las opiniones que se les ofrece de ellos, hasta tal punto que aceptan como adecuado para ellos un informe de personalidad standard, y dado que el trabajo de Eysenck (1947) incluye en su consideración todo tipo de sugestibilidad, mantendremos ambas hipótesis.

De acuerdo pues con el modelo de Eysenck, las hipótesis a someter a prueba en nuestro trabajo serán:

1. Los sujetos serán tanto más sugestionables (considerarán tanto más adecuado el informe para describir su personalidad), cuanto mayor sea su puntuación en $\mathbf{N}$.

2. Los sujetos con puntuaciones altas en $\mathrm{N}$ y $\mathrm{E}$, y los sujetos con puntuaciones bajas en $\mathrm{N}$ y E, serán menos susceptibles a la sugestión (considerarán menos adecuado el informe para describir su personalidad) que los sujetos con puntuaciones altas en $\mathrm{N}$ y bajas en $\mathrm{E}$, y que los sujetos con puntuaciones bajas en $\mathbf{N}$ y altas en $\mathrm{E}$.

\section{METODO}

\section{a) Sujetos:}

El trabajo se llevó a cabo con una muestra de 45 sujetos, 36 mujeres y 9 hombres, entre los estudiantes de $2 .^{\circ}$ curso de psicología que habían seguido durante un trimestre un curso dedicado al estudio de la psicología de la personalidad. Todos estos estudiantes habian realizado anteriormente, en dos sesiones diferentes, las siguientes pruebas de personalidad: E.P.I. (Eysenck y Eysenck, 1964), RII (Pelechano, 1975), C.E.P. (Pinillos, 1969), ya descritas, además de un cuestionario, BS (a), dirigido a medir una variable de reactividad situacional denominada «depresión situacional». No describiremos este cuestionario, del que se 


\section{Estudios}

incluye un ejemplar al final del experimento, dado que solamente se usó para poder dar una información mayor a los sujetos en el informe, sin provocar su desconfianza. Todos estos sujetos, además, habían realizado el experimento de trazado en aparatos, sobre el que también se hace referencia en el informe.

\section{b) Procedimiento:}

Se reunió a los sujetos que habían colaborado en un experimento anterior de trazado de aparatos (Labrador, 1980), y a los que se prometió que se los entregaría un informe personal con los resultados que habían obtenido a partir de los cuestionarios de personalidad que rellenaron para realizar ese experimento.

A todos los sujetos se les entregó un falso informe de personalidad, que habia sido preparado por el experimentador basándose esencialmente en el informe preparado para el experimento de Avia y Bragado (1975), por lo que el informe sólo casualmente podia tener alguna relación con las características personales de los sujetos.

El informe constaba de nueve enunciados que hacían referencia de forma muy concreta a los distintos aspectos de la personalidad que los alumnos sabían que podian medir los cuestionarios que habian rellenado. Un modelo del informe puede encontrarse al final de este experimento.

Una vez reunidos los sujetos en el aula de clase, se les indicó:

«Se os va a entregar el informe de personalidad que se os prometió cuando realizásteis los cuestionarios de personalidad. Dado que alguno de los cuestionarios usados están en período de adaptación y prueba, se os pide que, por favor, en una hoja aparte, deis una evaluación de hasta qué punto creéis que cada uno de los enunciados del informe os describe correctamente, de forma que si pensáis que es totalmente adecuado para describiros, le déis un valor de 10 , si no lo es tanto, valores inferiores, y si es completamente inadecuado le déis un valor de 0 . Como nos interesa vuestra opinión personal, es aconsejable que no comentéis nada del informe con otros compañeros, para que no pueda influiros en vuestras decisiones, hasta que no hayáis acabado la evaluación.

Tenéis todo el tiempo que necesitéis para llevar a cabo este trabajo.»

Después de estas instrucciones, se iba llamando personalmente a cada uno de los sujetos y se le entregaba un informe con su nombre, indicándole que se le entregaba una fotocopia del original que guardaba el experimentador para poder estudiar sus evaluaciones. Finalizada la tarea, se iba recogiendo a cada sujeto la hoja de evaluación conforme acababa, indicando que guardaran silencio para no molestar a los demás.

\section{c) Análisis de datos:}

Se tomó de cada uno de los sujetos la media de las nueve puntuaciones correspondientes a cada uno de los enunciados y se pasó a analizar estas medias por grupos formados a partir de las distintas variables de personalidad consideradas.

\section{RESULTADOS}

Se llevó a cabo primero un análisis de los resultados en general, y después considerando el sexo y los efectos de cada variable de personalidad de manera aislada. Los resultados pueden verse en la tabla n. ${ }^{\circ} 1$.

Como puede apreciarse, el resultado promedio del acuerdo de los sujetos con el informe es de 7.87, que indica claramente que se ha producido un efecto bastante intenso de sugestión, y que en general los 
TABLA I

Puntuaciones de grado de acuerdo con el Informe de Personalidad en los distintos grupos formados a partir de las puntuaciones de los sujetos en los distintos cuestionarios.

\begin{tabular}{|c|c|c|c|c|}
\hline & $\mathbf{N}$ & $\bar{x}$ & $S x$ & Novel de Significación \\
\hline 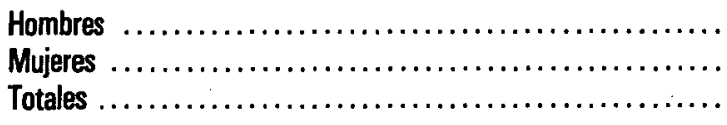 & $\begin{array}{r}9 \\
36 \\
45\end{array}$ & $\begin{array}{l}7.848 \\
7.932 \\
7.872\end{array}$ & $\begin{array}{l}0.676 \\
1.074 \\
1.025\end{array}$ & \\
\hline 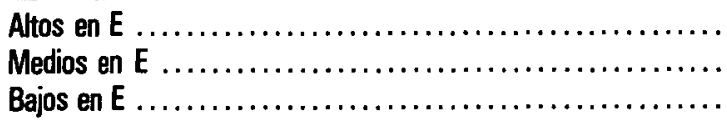 & $\begin{array}{l}14 \\
19 \\
12\end{array}$ & $\begin{array}{l}7.723 \\
7.902 \\
7.996\end{array}$ & $\begin{array}{l}1.042 \\
1.211 \\
0.548\end{array}$ & \\
\hline 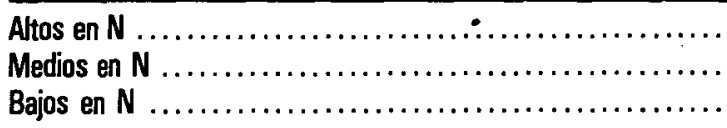 & $\begin{array}{r}9 \\
23 \\
12\end{array}$ & $\begin{array}{l}8.514 \\
7.585 \\
8.098\end{array}$ & $\begin{array}{l}0.729 \\
1.112 \\
0.5996\end{array}$ & $>\mathrm{t}=2.26, \mathrm{p}$ \\
\hline 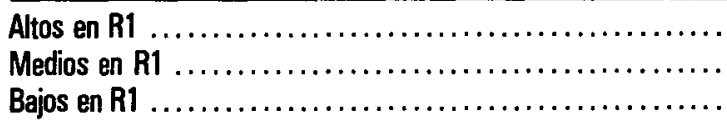 & $\begin{array}{r}9 \\
23 \\
12\end{array}$ & $\begin{array}{l}7.638 \\
8.059 \\
7.847\end{array}$ & $\begin{array}{l}1.206 \\
1.057 \\
0.558\end{array}$ & \\
\hline 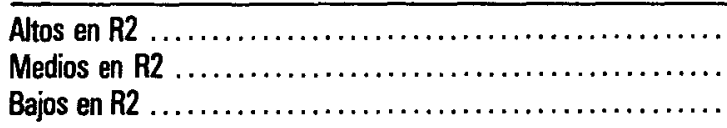 & $\begin{array}{r}9 \\
21 \\
14\end{array}$ & $\begin{array}{l}7.674 \\
8.075 \\
7.8292\end{array}$ & $\begin{array}{l}1.268 \\
1.268 \\
0.701\end{array}$ & \\
\hline 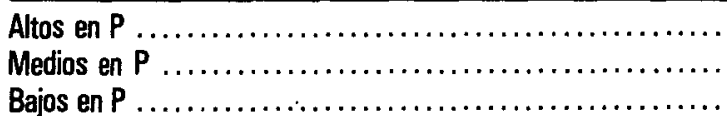 & $\begin{array}{r}13 \\
22 \\
9\end{array}$ & $\begin{array}{l}8.012 \\
8.077 \\
7.378\end{array}$ & $\begin{array}{l}0.836 \\
1.108 \\
0.693\end{array}$ & \\
\hline
\end{tabular}

sujetos aceptaron con un valor bastante elevado el informe presentado.

No aparecen diferencias en función del la variable sexo, como por otro lado era de esperar de acuerdo con los resultados obtenidos en los trabajos anteriores, así como por el pequeño número de sujetos varones en la muestra.

De las variables de personalidad, la variable E-I no presenta diferencias significativas entre los distintos grupos, tal como podría esperarse a partir de las consideraciones de Eysenck, pero sí la variable $\mathrm{N}$, y además estas diferencias aparecen en la dirección prevista por la hipótesis 1 de Eysenck, los sujetos con valor alto en $\mathbf{N}$ presentan un acuerdo mayor con el informe, que los sujetos con valores medios en $\mathrm{N}(\mathrm{p}<.05)$, y que los sujetos con valores bajos en $\mathbf{N}$ (la diferencia en este caso no alcanza a ser significativa).
Así pues, parece relativamente confirmado que los sujetos con valores altos en $\mathrm{N}$ son los más sugestionables, pero la hipótesis de Eysenck de 1947, que propone una correlación lineal y positiva entre $\mathrm{N}$ y grado de sugestionabilidad, no se confirma totalmente en cuanto que las relaciones son curvilíneas.

Pasemos a considerar la hipótesis 2, para la cual debemos dividir a los sujetos en grupos considerando conjuntamente los valores en $\mathbf{N}$ y $\mathbf{E}$. Se formaron los siguientes grupos en función de sus puntuaciones en el E.P.I.:

-NE.-Sujetos con puntuaciones iguales o superiores al percentil 70 en las dimensiones $\mathrm{N}$ y $\mathrm{E}$.

-NI.-Sujetos con puntuaciones iguales o superiores al percentil 70 en la 


\section{Estudios}

dimensión $\mathrm{Ne}$ iguales o inferiores al de $\mathbf{3 0}$ en la de E.

-CE.-Sujetos con puntuaciones iguales o inferiores al percentil 30 en N, e iguales o superiores al 70 en $\mathbf{E}$.
CI.-Sujetos con puntuaciones iguales $o$ inferiores al percentil 30 en $\mathrm{N}$ y $\mathrm{E}$.

Los resultados obtenidos por este grupo pueden verse en la tabla $n .{ }^{\circ} 2$.

\section{TABLA II}

Diferencias entre Medias de los grupos CE, Cl, NE y NI.

Hombres

\begin{tabular}{ccccc}
$\mathrm{N}$ & $\bar{x}$ & $\mathrm{Sx}$ & rlaceptación f1) & raceptación $f 2$ \\
\hline 9 & 7.848 & 0.676 & .2068 & .0516 \\
36 & 7.932 & 1.074 & .0399 & .100
\end{tabular}

Como puede observarse en esta tabla, los resultados aparecen en la dirección formulada por la hipótesis, los sujetos NE, son los menos susceptibles a la sugestión, y los NI, son los que más, invirtiéndose las relaciones de los sujetos que puntúan bajo en $\mathrm{N}$, donde los extravertidos aparecen como más sugestionables que los introvertidos. Además no sólo aparecen los resultados en la dirección esperada, sino que en algunos casos las diferen- cias entre los grupos son significativas, en concreto, las diferencias entre los grupos NI y NE $(p<.05)$, y la diferencia entre los grupos CI y NI $(\mathrm{p}<.01)$.

No obstante hay que indicar que no aparece una diferencia tan importante entre los grupos CE y CI, como era de esperar para que pudiera aparecer la distribución propuesta por Furneaux (1961) o por Eysenck (1966 y 1967). Ver figura n. ${ }^{\circ} 4$.

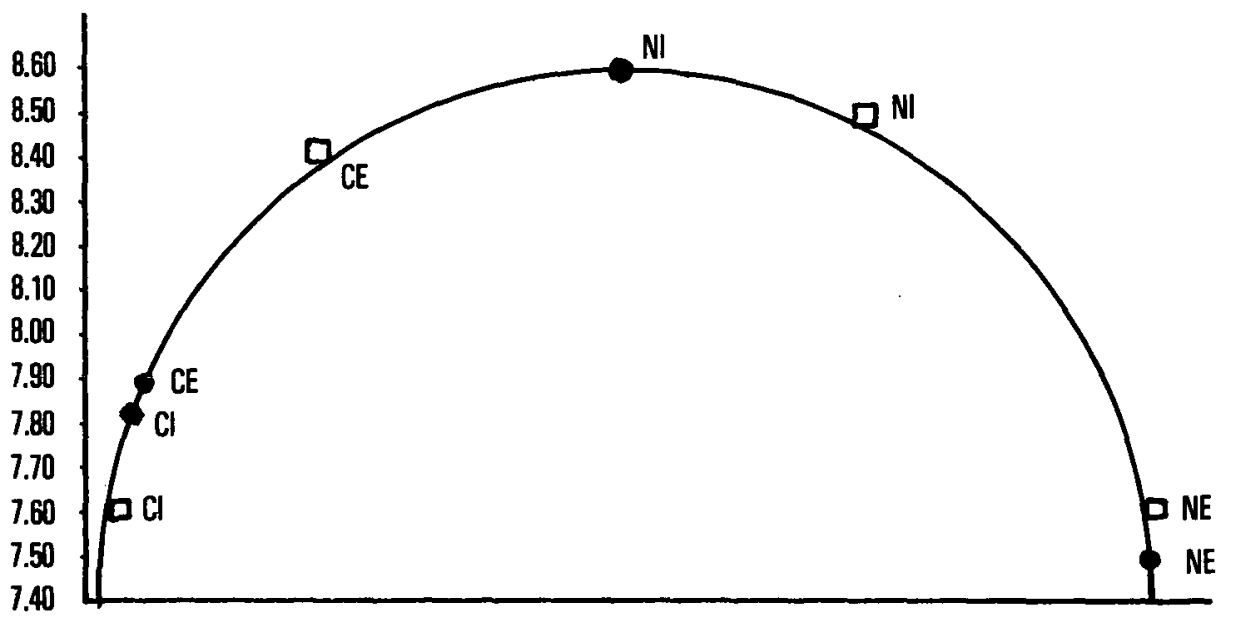

Figura 4.-Posiciones reales obtenidas por los sujetos ( $($ ) y posiciones que teóricamente deblan obtener $\square$, según Eysenck.

Con respecto a los factores de rigidez, dado que no apareció ninguna diferencia entre los grupos, y dado que en el trabajo de Avia y Bragado (1975), las diferencias aparecieron al tener en cuenta el sexo de los sujetos (pues aparecían correlaciones 
de distinto signo para cada uno de los sexos, en especial en el factor FI) se llevó a cabo un análisis correlacional por separa- do para cada sexo, tanto en el F1, como en el F2, los resultados pueden verse en la tabla $n .^{\circ} 3$.

\section{TABLA III}

Diferencias en realización de los sujetos seprados por sexos y su correlación con los factores F1 y F2.

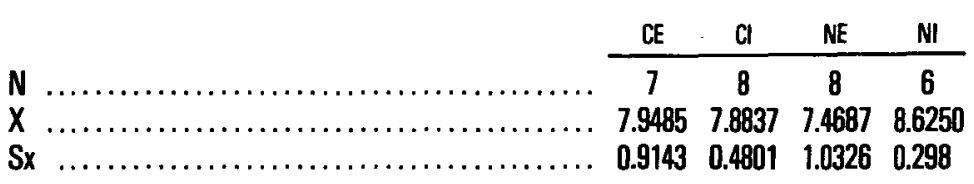

Diferencias Significativas NE-Nl: $t=2.464 \quad 2.179 . \quad p<.05$

$\mathrm{Cl} \cdot \mathrm{Nl}: \mathrm{t}=3.089 \quad 3.055 \quad \mathrm{p}<.01$.

Como puede apreciarse en esta tabla, el F1 vuelve a presentar una ligera correlación positiva con el grado de aceptación del informe en los hombres, pero en las mujeres la correlación aunque prácticamente nula, tiene signo negativo. No obstante, ninguna de estas correlaciones es significativa ni está próxima a serlo. El F2, tiene aún menor capacidad para explicar la aceptación del informe, pues prácticamente no aparecen correlaciones.

Tampoco la variable de paranoidismo es capaz de diferenciar el grado de aceptación del informe, aun cuando los sujetos con valores bajos en paranoidismo presentan unos valores inferiores.

\section{COMENTARIOS}

Como se esperaba volvió a aparecer el fenómeno de aceptación del informe standard de personalidad. La mera aparición de este fenómeno es ya un dato a favor de los modelos derivados del análisis funcional, en cuanto que conociendo el tipo de estímulos a que se somete el sujeto, sea cual sea la personalidad de éste, van a aparecer unas conductas (en este caso aceptación del informe) indicadoras de la existencia de este afecto de sugestión. No obstante, es cierto que esta afirmación es un poco general y hasta cierto punto ambigua, en cuanto que se debía aclarar cuál de las condiciones estimulares o de la interacción entre estas son las que realmente intervienen en la producción de este efecto. Así pues, aunque se pensó en un primer momento que una de las condiciones estimulares fundamentales era el prestigio y seriedad del experimentador, los trabajos de Ulrich, Stachnick y Staiton (1963) o de Snyder y Larson (1972), en los que los experimentadores eran los mismos estudiantes y volvió a aparecer este último efecto, demostraron que esta variable no era $\tan$ fundamental como parecía.

Otras alternativas explicativas, son las de la importancia de la deseabilidad social de los enunciados, comentada ya por Edward (1957), o las simples tendencias a dar respuestas afirmativas a los enunciados, tan frecuentemente criticada en lo que se refiere a los items de los cuestionarios de personalidad (Mischel, 1968), que en definitiva guardan un parecido bastante grande con éstos.

Así pues, en este experimento, el modelo de personalidad de Eysenck es más correcto y útil para hacer predicciones sobre el comportamiento de los sujetos que otras alternativas, como pueden ser 


\section{Estudios}

en concreto las de los modelos derivados del análisis funcional.

Ciertamente puede ser un tanto cuestionable la explicación de cómo reformula esta predicción y hasta qué punto puede decirse que esta predicción se fundamente completamente en el modelo de Eysenck. Por otro lado, el hecho de que por las características de la prueba la relación entre el experimentador y el sujeto se viera muy disminuida con respecto a las típicas pruebas de susceptibilidad en el test de balanceo corporal, dado que en este caso el sujeto realizaba su tarea de forma individual, sin relación directa, no justificaría al menos según el desarrollo propuesto por Furneaux (1961), el que se sumara al nivel de $\mathrm{N}$ alto del $\mathrm{NE}$ la intensa producción de drive que se espera del extrovertido en una relación interpersonal. No obstante, el que los resultados aparezcan en la dirección postulada (aunque ciertamente no siempre con valores suficientes para hacer significativas las diferencias entre grupos) y que esto mismo haya aparecido en otros trabajos, como el de Gibson y Corcoran (1975), o el de Gibson, Corcoran y Curran (1977), donde este efecto de la interacción entre la variable de E-I y la condición estimular del experimento hagan también algo difícil al aplicar la explicación desarrollada por Furneaux (1961), nos lleva a pensar que el problema no está tanto en lo correcto de las predicciones, sino en la explicación del porqué de estas predicciones.

En resumen pues, parece ser que este experimento presenta un pequeño apoyo al modelo de Eysenck, y lo que es más interesante, este apoyo, aún siendo pequeño por la falta de significación en algunos resultados, aparece en tareas que implican la participación en funciones superiores, y por lo tanto más complejas y más interesantes que las meras tareas de repeticiones motoras simples. Evidentemente seria muy interesante que fuera en las tareas donde se exige de manera más completa y específica la participación de las funciones superiores, donde las predicciones a partir de los modelos de personalidad sean más eficaces, lo que implicaría que sería suficiente con hacer algunas distinciones adicionales sobre los sistemas de respuestas más simples del organismo (como las respuestas motoras), para poder realizar predicciones más acertadas también en esas tareas.

Antes de concluir convendría hacer un comentario sobre la falta de efectos encontrados en las variables de rigidez, en concreto el factor F1, que sí había servido para diferenciar, aunque con valores bajos, los resultados de los sujetos, separados por sexos, en el trabajo de Avia y Bragado (1975). En nuestro trabajo han aparecido de nuevo diferencias en el grado de aceptación del informe en función de la variable $F 1$, al separar a los sujetos en función del sexo, pero a pesar de que los resultados aparecen en la dirección especificada por estas investigadoras, no son ni mucho menos significativas, si bien puede deberse en gran parte al escaso número de sujetos varones de nuestra muestra. En cualquier caso no está claro el por qué aparecían estas diferencias. 


\section{Estudios}

\section{INFORME PSICOLOGICO DE LOS RASGOS CONSTITUTIVOS DE LA PERSO- NALIDAD EN FUNCION DE LAS PUNTUACIONES ALCANZADAS A PARTIR DE LAS PRUEBAS REALIZADAS.}

1.-Neuroticismo: Ha obtenido puntuaciones moderadamente bajas, significativas de un control bastante alto de la emocionalidad ante las situaciones de stress.

2.-Extraversión: Ligeramente introvertido. En las situaciones sociales suele costarle entrar en interacción con los demás, pero sin embargo, una vez pasados los primeros momentos, la interacción suele tener un grado aceptable.

3.-Principalismo social: Sus puntuaciones en este factor sugieren que no suele creer sin sentido crítico en las grandes palabras tales como justicia, patria y sentido del deber. Se presenta más bien como una persona flexible dispuesta a escuchar e incluso' tener en cuenta opiniones ajenas a las suyas.

4.-Valoración del trabajo: Sus respuéstas en lo que se refiere a este factor le presentan, en relación a la media de la población, como algo más exigente que en el caso anterior. Valora positivamente el trabajo y se presenta poco flexible ante la realización de tareas concretas sin perder el sentido de la realidad que le circunda: en suma, ligeramente superior a la media en la importancia que da al trabajo y con valoración francamente positiva del mismo.

5.-Conformismo en las relaciones interpersonales: Puntúa por debajo de la media (y la diferencia es estadísticamente significativa). Parece ser inconformista en las relaciones personales con un criterio demasiado exigente por lo que se refiere a la selección de sus amistades. Es probable que haya influido la edad en estas puntuaciones.

6.-Conformismo social: En este factor ocurre un fenómeno similar al caso anterior. Posee un sentido crítico quizá excesivo para las condiciones sociales que le rodean. Independientemente, claro está, de que las condiciones sociales no sean las óptimas, su imagen de la sociedad española actual es negativa.

7.-Aislacionismo social: En este factor, que también tiene en cuénta la dureza en las relaciones personales, sus resultados sugieren que es capaz de comprender la acción de sus semejantes pero que no es capaz de comprender sus intereses personales.

8.-Depresión situacional: Sus puntuaciones indican que percibió la prueba de laboratorio como relativamente fácil, independientemente de las indicaciones que le hacía el experimentador.

9.-Depresión reactiva: Sus puntuaciones sugieren que a lo largo del día pasa por una serie de cambios de humor que forman ciclos de euforia-depresión. Los cambios observados no son, sin embargo, suficientemente importantes como para ser tomados en consideración. 


\section{Estudios}

APELLIDOS .

ITOMRRE

Debajo de estas Ilneas encontrará una serie do callfication y sus -opuestos.Se trata de decidir -sin pensar demeslado- cuál de las dos pe-labras do cada fila erpresa mejor su estado actunl o el modo en que se siente ahora. Una vez decílido, haga una cruz en la columna SI de la hoje de respuestas cuando escoja la palabra de la 1zquierda, $\bar{y}$ en la de la seluma vo cuando lo sea por la de la derecha, en el supuesto de no decidirse porrninguna, deje on blanco la cabilla correspondiente al par cie Fa labras-

\section{IZQUIERDA}

\section{1.- fresco}

R.- Indiferente

3.- - otlórico

4.- 1leno de Exito

5.- Irritado

6. - indeciso

7.- alegro

8.- de buen humor

9.- $\sin$ apetito

10.- sociablo

11.- sin aprecto de nadie

12.- relajado

13.- fellz

14.- tímido

15.- Inpuro

2C.- Beguro

17.- at andonado

18.- equilibrado

19.- seguró do si mitumo

20. - desdichado

21. - flextblo

22. - cansado

23. - Indec18o

24. - tranguilo

25.- apát1co

26.- Initil

27.- torpe

28.- supertor
DERECEA

1.- agotado

2.- Interesado

3.- melancblico

4.- fracasado

5.- so segado

6.- dec1dido

7.- pesaroso

8. - contrariado

9.- con apetito

10.- insociable

11.:- npreciado.

15. - on tonsión

13.- desgraciado

14. - atrovido

15.- puro

16.- amenazado

17.- socorrido

18. - agitado

19. - inseguro

20.- dichoso

21.- rigido

22.- descansndo

23.- docids.do

24. - Intranguilo

25.- energico

26. - Indispionsablo

27.- Imaginativo

28.- Infartor 


\section{Referencias}

M. D. Y BRAGADO, M. C.: Los informes de personalidad y la credulidad ante los test: un efecto palcebo Anál. y Modif. de Cond., 1975, 1, 33-54.

EDWARDS, A. L.: The social desirability variable in personality assessment and research. Nueva York, Dryden, 1957.

EYSENCK, H. J.: The biological basis of personality. Springfield (Illinois), Ch. C. Thomas, 1967.

EYSENCK, H. J.: Personality and experimental psychology. Bull. Brit. Psychol. Soc., 1966, 62, 1-28.

EYSENCK, H. J. y EYSENCK, S. B. G.: Manual of the Eysenck Personality Inventory. Londres, Universidad de Londres, 1964. (Adaptación española T.E.A., 1973).

FURNEAUX, W. D.: Neuroticism, extraversion, drive and sugestibility. Internat. J. Clin. Exper. Hypnosis, $1961,9,195-214$.

FURNEAUX, W. D.: Neuroticism, extraversion and sugestibility: a coment. Internat. J. Clin. Exper. Hypnosis, 1963, 11, 201-202.

FURNEAUX, W. D. y GIBSON, H. B.: The Maudsley Personality Inventory as a predictor of susceptibility to hypnosis. Internat. J. Clin. Exper. Hypnosis, 1961, 9, 167-177.

GHISELLI, E. E.: Moderating effects and differencial reability and validity. J. Appl. Psychol., 1963, 47, 81-86.

GIBSON, H. B. y CORCORAN, M. E.: Personality and differential susceptibility to hypnosis: further replication and sex diferences. Brit. J. Psychol., 1975, 66, 513-520.

GIBSON, H. B.; CORCORAN, M. E. y CURRAN, J. D.: Hypnotic susceptibility and personality: the consequences od diazepam and the sex of the subjects. Brit. J. Psycol., 1977, 68, 51-59.

GIBSON, H.B. y CURRAN, J. D.: Hyptonic susceptibility and personality: a replication study. Brit. J. Psychol., 1974, 65, 283-291.

HILGARD, E. R.: Hypnotic susceptibility. Nueva York, Harcourt, 1965.

HILGARD, E. R. y BENTLER, P. M.: Predicting Hypnotizability from the M.P.I. Brit. J. Psychol., 1963, $54,63-69$.

LABRADOR, F. J.: Estudio de la utilidad de los modelos factoriales-biológicos de personalidad: Eysenck y Gray. Madrid, Universidad Complutense, Tesis Doctoral no publicada, 1980.

MISCHEL, W.: Personality and assessment. Nueva York, Wyley, 1968.

PELECHANO, V.: Psicología estimular y modulacion. Madrid, Marova, 1975.

PINILLOS, J. L.: Cuestionario de personalidad del C.E.P., manual. Madrid, T.E.A., 1969 (2." edición).

SNYDER, C. R. y LARSON, G. R.: A further look at student acceptance of general personality interpretations. J. Couns. Clin. Psychol., 1972, 38, 384-388.

ULRICH, R. E.; STACHNIK, T. J. y STAITON, N. R.: Student acceptance of generalized personality interpretations. Psychol. Rep. 1963, 13, 831-834.

\section{Resumen}

Se presento a los sujetos un informe estándar sobre su personalidad para estudiar hasta qué punto lo acaptaban como adecuado para describirlos a ellos mismos. Se predecía que el grado de aceptación de estos informes variaria en función de las características de personalidad de los sujetos. De acuerdo con Eysenck (1967), los sujetos con valores máximos en $\mathrm{E}$ y N a la vez (NE y $\mathrm{Cl}$ ) serian los menos sugestionables, mientras que los sujetos con valores altos en una dimension y bajos en la otra (NI y CE) serían los más sugestionables. Los resultados apoyan ligeramente esta hipótesis. No obstante se cuestiona el que las predicciones se deriven realmente del modelo de Eysenk y no sean formulaciones «ad hoc".

\section{Summary}

A standard personality inform was presented to the subjects to study as much as it is accepted as a correct description of theyself. Accord to the prediction, the.degree of acceptation of such inform must varie in fuction to personality subject's characteristics. In agreement with the Eysenck (1967) model the subjects with high and small values in both, $\mathrm{E}$ and $\mathrm{N}$, dimensions ( $\mathrm{NE}$ and $\mathrm{CI}$ ) must be the less suggestibles, whereas the subjects with high value in one dimension and small value in the other (NI and CE) must be the suggestiblests. The result in part support these predictions. However the fact that the predictions were really derivated of the Eysenck's model is questioned. 


\section{Resumé}

On a presenté aux sujets un texto standard sur sa personnalité por étudier jusqu'a a quel point ils pouvaient l'accepter comme adapté à la description d'eux-meme. On pouvait s'attendre à ce que le degré d'acceptation de ces textes variraient en fonction des caracteristiques des personnalites des sujets. En accord avec Eysenck (1967) les sujets ayant des valeurs maxima et minima en E et $N$ (NE et $\mathrm{CI}$ ) seraient les moins influencts, alors que les sujects ayant valeur eleve pour une dimension et basse por l'autre seraient les plus influencés. Les resultants confirment légèrement ces prèdictions. Cependant on serait tenté de douter que les predictions soient réalement issues du modèle de Eysenck, mais plutôt formulées «ad hoc». 